\title{
Bergsoniana
}

1 | 2021

Reassessing Bergson

\section{Bergson et la télépathie : à propos d'une correspondance inédite}

Renaud Evrard

\section{(2) OpenEdition}

\section{Journals}

Édition électronique

URL : https://journals.openedition.org/bergsoniana/463

DOI : 10.4000/bergsoniana.463

ISSN : 2800-874X

Éditeur

Société des amis de Bergson

\section{Référence électronique}

Renaud Evrard, «Bergson et la télépathie : à propos d'une correspondance inédite », Bergsoniana [En ligne], 1 | 2021, mis en ligne le 01 juillet 2021, consulté le 08 novembre 2021. URL : http:// journals.openedition.org/bergsoniana/463; DOI : https://doi.org/10.4000/bergsoniana.463

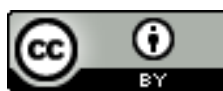

Les contenus de la revue sont mis à disposition selon les termes de la Licence Creative Commons Attribution 4.0 International. 


\title{
BERGSONET LA TÉLÉPATHIE : À PROPOS D'UNE CORRESPONDANCE INÉDITE
}

\author{
Renaud EVRARD
}

\section{L'investissement initial de Bergson dans les recherches psychiques}

À 27 ans, alors qu'il est professeur de philosophie à Clermont-Ferrand, Bergson participe aux expériences qui ont lieu chez le docteur Moutin avec des sujets en état d'hypnose. Il publie en 1886, dans la Revue philosophique de la France et de l'étranger (Bergson 1886a), les résultats de ces expérimentations portant sur l'hyperesthésie (des capacités perceptives exacerbées) et la simulation inconsciente, ce qui lui vaudra d'ailleurs une réprimande de l'Inspection générale del'enseignement supérieur. Des jeunes gens sont allongés sur un canapé situé à quatre mètres de l'hypnotiseur. Celui-ci se tient debout devant eux et ouvre un livre au hasard dont ils ne peuvent voir que la couverture. Il le tient verticalement à dix centimètres environ de ses yeux, mais légèrement en dessous, de manière à pouvoir en même temps fixer par le regard le sujet endormi. Or l'un des sujets parvient à lire des lignes entières du texte. Plutôt que d'invoquer la transmission de pensée, Bergson suggère alors qu'il s'agit d'une hyperesthésie visuelle : le sujet serait parvenu à lire le livre en distinguant le reflet des lettres dans l'œil de l'hypnotiseur.

Cependant le projet véritable de cette recherche est fondamentalement dissimulé dans son format final, comme Bergson s'en confie à son correspondant britannique, Frederic W. H. Myers (1841-1903), fondateur de la Society for Psychical Research (SPR) en Angleterre et inventeur en 1882 du terme "télépathie " (Luckhurst 2002), définie comme la 
" communication d'impression de toute espèce d'un esprit à un autre de manière indépendante des voies sensorielles reconnues " (Myers 1903, xxii). ${ }^{1}$ Dans une lettre datée du $1^{\text {er }}$ décembre 1886 , Bergson avoue à ce dernier " qu'ayant abordé ces études avec une arrière-croyance à la suggestion mentale et à la transmission de la pensée, ayant essayé d'y façonner mes sujets et de les amener, petit à petit, à deviner les mots ou les images que je me représentais, je n'ai jamais pu obtenir avec eux le moindre résultat " (Bergson 1886b). ${ }^{2}$ C'est donc bien dans le cadre d'une recherche sur la télépathie que Bergson débute son étude. Et s'il fait cet aveu, c'est pour contredire l'hypothèse soumise par Myers selon laquelle ses sujets hypnotisés seraient parvenus à percevoir des stimuli minuscules en puisant directement dans l'esprit de l'expérimentateur.

Dans la même lettre, Bergson se dit familier des travaux de Myers « depuis un certain temps déjà. " Il se nourrit également du " très remarquable article sur la suggestion mentale » (Bergson 1886a, 62) du physiologiste et futur prix Nobel Charles Richet, récemment publié dans la même revue (Richet 1884). En introduisant cette possibilité d'une hyperesthésie visuelle, Bergson est venu suggérer que toutes les précautions n'avaient pas été prises dans les expériences de Richet : ses sujets auraient pu percevoir les cartes à deviner se reflétant sur sa cornée. Toutefois, dans la suite de sa correspondance avec Myers (20 janvier 1887), Bergson admet que "l'hypothèse d'une 'thought-transference', il est vrai, n'est pas tout à fait écartée, mais elle est tellement improbable qu'il n'y aurait pas lieu, vous le dites vous-même, de 'press the case' de ce côté " (Bergson 1887). Puis, accusant réception des Proceedings de la SPR, il exprime son intérêt pour ce « nombre si considérable de faits [observés] d'une manière scientifique » et exprime son humilité : «Le jour où vous pourrez tirer de tout cela quelques vues $^{3}$ systématiques, il faudra renoncer sans doute à bien des idées reçues sur les rapports de la pensée avec le système nerveux ; mais je ne vois aucune raison pour nier à priori des phénomènes de ce genre " (Bergson 1887). S’il rechigne à expliquer ces phénomènes autrement que par une transmission de la pensée, il en conçoit la possibilité : «Il faudra sans doute se résigner à en venir là. » Myers (1887a) fit écho à l'article de Bergson dans une note publiée en janvier 1887 dans la très respectable revue Mind,

1. Traduction de : " the communication of impressions of any kind from one mind to another, independently of the recognized channels of sense. "

2. Je remercie Andreas Sommer de m'avoir communiqué ces lettres.

3. Régine Plas, qui cite ces lettres, a erronément remplacé le mot «vues " par "idées " (Plas 2000, 115). 
considérée comme le premier journal de langue anglaise ayant diffusé des travaux de psychologie expérimentale (Nicolas et al. 2000), note reprise peu après, mot pour mot, dans les Proceedings de la SPR (Myers, 1887b), en se référant à d'autres expériences récentes dues à d'autres auteurs français. Selon Thibaud Trochu (2014, 209), William James découvrira Bergson par ce biais et le citera dans ses Principles of Psychology (James 1890, 609). De façon posthume, un ouvrage de Myers déploiera cette théorisation systématique attendue par Bergson (Myers 1903 ; Kelly et al. 2007).

Que la télépathie expérimentale soit un point de départ de la philosophie bergsonienne a de quoi surprendre, tant on a l'impression, à lire les commentateurs de son œuvre, «que l'intérêt de Bergson pour la télépathie et les questions connexes n'est que périphérique " (Méheust 1999, 241). Il a fallu attendre l'article bien documenté de Camille Pernot $(1964,17)$ pour que soit affirmé « que la 'science psychique' fut l'objet d'un intérêt constant de la part de Bergson et qu'elle trouve, dans sa doctrine et ses conceptions méthodologiques, des justifications profondes, les plus profondes, sans doute, qu'on lui ait jamais fournies. " Le canadien Robert C. Grogin $(1988,65)$ affirme avoir recensé près de cinq mille articles consacrés au philosophe, et n'avoir trouvé que ce seul article abordant directement cette thématique. Encore faut-il noter que Pernot tient un discours qui, tout en soulignant la grande convergence entre le spiritualisme bergsonien et la " recherche psychique, » vient disqualifier ironiquement que le recours aux médiums soit proposé en définitive par Bergson (1932) comme la seule technique efficace pour résoudre les problèmes moraux posés par notre civilisation (Pernot 1964, 18). Ce discrédit est d'ailleurs la position la plus fréquemment rencontrée dans la littérature. Karl Sarafidis $(2009,76)$ s'en fait l'écho lorsqu'il écrit : "Nous ne pouvons feindre d'ignorer l'intérêt constant de Bergson pour ce qui ne constitue à nos yeux que de pures fictions de positivité scientifique relevant davantage de la superstition populaire que de l'observation empirique. "Toutefois, depuis le travail d'édition et de contextualisation des textes réalisé par l'équipe de l'édition critique des Presses universitaires de France dès la fin des années 2000, ce dossier des liens entre Bergson et les recherches psychiques est désormais mieux appréhendé. 


\section{Empreinte des recherches psychiques sur l'œuvre de Bergson}

L'analyse de l'œuvre bergsonienne à la lumière des recherches psychiques ayant seulement été ébauchée par quelques auteurs (Méheust 1999, 240 255; Fujita 2007), nous nous bornerons à en rassembler quelques indices.

Matière et mémoire (Bergson 1896) ne traite pas directement de recherche psychique, cependant plusieurs « psychistes " font remarquer à quel point ce livre peut être lu comme une contribution à l'étude des questions de télépathie et de survie de l'esprit après la mort du corps (Grogin 1988, 56-57 ; Price 1941 ; Méheust 1999, 244 ; van Dongen 2014). La théorie bergsonienne des rapports de l'esprit et du corps permet de légitimer les recherches psychiques, en critiquant la thèse psychophysiologique selon laquelle la perception serait produite par le système nerveux. "Cette théorie montre ainsi qu'une extension des facultés de percevoir est possible, sans extension concomitante de la machine corporelle " (Madelrieux et al. 2009, 288). Pernot (1964, 10, 12 et 13) souligne à plusieurs reprises la convergence voire même la solidarité entre la thèse de Matière et mémoire et les arguments de Bergson en faveur de la " recherche psychique. "Bergson emploie dans cet essai - ce qui deviendra récurrent par la suite - les cas d'exaltation de la mémoire dans les expériences de mort imminente comme " les faits les plus instructifs venant soutenir sa théorie des deux mémoires indépendantes " (Evrard 2018).

L'investissement de Bergson ne se fait pas qu'au travers de ses écrits, mais aussi dans ses engagements institutionnels en faveur de la recherche psychique. En 1900, Bergson devient un membre de la première heure du comité exécutif de l'Institut général psychologique (IGP), l'une des premières sociétés de psychologie en France avant la "rupture " avec la "parapsychologie» (cf. Plas 2000 ; Evrard 2016, chapitre 6). Sa conférence sur "Le rêve " du 16 mars 1901 pointe, à la toute fin de son exposé, vers l'horizon des recherches psychiques puisqu'il appelle la psychologie à diriger son effort vers l'étude du sommeil profond, pour y « scruter ces phénomènes plus mystérieux qui relèvent de la 'recherche psychique'. » Dans la version initiale du texte (publiée dans la Revue scientifique dirigée par Richet, puis dans Mélanges) transparaît un enthousiasme qui sera légèrement édulcorée dans la version publiée dans L'Energie spirituelle (Bergson 2017), ce qui peut conduire à s'interroger sur une "duplicité » (Sarafidis 2009) de Bergson. Le philosophe ouvre vers l'étude des rêves télépathiques tout en restant en recul : 
Je n'ose me prononcer sur les phénomènes de cet ordre, mais je ne puis m'empêcher d'attacher quelque importance aux observations recueillies avec une méthode si rigoureuse et un si infatigable zèle par la Society for Psychical Research [...]. Si la télépathie influençait nos rêves, c'est vraisemblablement dans ce sommeil très profond qu'elle aurait le plus de chance de se manifester (Bergson 1972, 462).

Cette prudence est peu goûtée par les partisans des sciences psychiques présents à sa conférence. L'un d'entre eux, le colonel de Rochas, exprime sa frustration dans l'après-coup :

Ce titre prometteur avait attiré un grand nombre de personnes qui, pendant une heure et demie, ont attendu que l'orateur, M. Bergson, sortît de la psychologie classique pour faire connaître les documents nouveaux fournis par la science psychique. Le savant professeur du Collège de France n’a pas eu cette audace et sa conférence, d'ailleurs charmante, s'est terminée juste au point où son auditoire espérait la voir commencer (de Rochas 1901).

En 1901, Bergson intègre le Groupe d'études des phénomènes psychiques de l'IGP, où il prend en 1905 la tête de la commission d'étude du médium à effets physiques Eusapia Palladino, avant de laisser la place à Pierre Curie et Arsène d'Arsonval (Bergson 1972, 673-674). Plus qu'un simple observateur, il participe activement aux contrôles expérimentaux (Méheust 1999, 242). D’après Méheust :

Il n'assiste qu'à six séances, mais a la chance d'observer toute la gamme des phénomènes allégués, et en sort perplexe, partagé, convaincu à la fois que le médium a parfois cherché à tricher, et que certaines de ses manifestations étaient d'une nature si extraordinaire qu'elles défiaient toute explication rationnelle (1999, 242).

Ce constat, qui est celui émanant du rapport final (Courtier 1908), se trouve plutôt énoncé chez Bergson comme relatif à l'impossibilité de prouver l'inexistence de quelque chose (Benrubi 1942, 21 ; Bergson 2017). Au sujet de la médium Leonora Piper, dont James a étudié les prouesses en matière de médiumnité intellectuelle, Bergson repèrera cette difficulté à établir des certitudes dans ce domaine : "Il intervient dans toutes ces expériences quelque chose de fuyant qui déconcerte l'esprit " (Bergson 2002, 302-303 ; Bergson 2011, 760).

Plusieurs témoignages attestent d'une conviction grandissante relative aux preuves expérimentales des phénomènes dits psychiques, dont celui d'Isaac Benrubi, cité par Grogin $(1988,51)$. «Toujours prudent dans ses conclusions, il affiche cependant de plus en plus son engagement en faveur de telles recherches" (Madelrieux et al. 2009, 274) et devient membre 
correspondant de la SPR en 1909. Fait rare, Bergson a accordé en 1910 un entretien à Georges Meunier, venu l'interroger sur ces phénomènes, dans lequel il confirme ses bonnes dispositions à l'égard des phénomènes physiques de la médiumnité et les élargit à la télépathie. Bergson "s'avoue ébranlé par la masse des faits qui paraissent témoigner de son existence. Il ne l'enseigne pas dans ses cours parce qu'il se sent tenu de n'enseigner que le certain ; mais il reconnaît que les témoignages accumulés sur cette faculté présumée font pencher la balance de son côté " (Méheust 1999, 243). Selon ses propres termes : "Après avoir pris connaissance des documents publiés depuis 25 ans sur ce sujet, déclare-t-il, j'estime pour ma part qu'il y a plus de probabilité en faveur de l'hypothèse de la télépathie qu'il n'y en a en sens contraire. S'il fallait parier pour ou contre, je parierais pour, sans hésitation. " (Bergson in Meunier 1910, 95). Il encourage néanmoins à la suspension de jugement car ces seules expérimentations auxquelles il a participé n'ont pas suffi à emporter son adhésion (Pernot 1964, 6).

En 1913, il est élu pour l'année président de la SPR et prononce à cette occasion un discours intitulé "'Fantômes de vivants' et 'recherche psychique' " qui a été considéré comme le "Discours de la méthode des sciences psychiques qualitatives " (Méheust 2005). ${ }^{4}$ "Il s'agit pour lui [...] de montrer que les préventions à l'encontre du projet même de la recherche psychique sont injustifiées et qu'elle incarne au contraire l'avenir de la science de l'esprit " (Madelrieux et al. 2009, 275), avis qui était déjà celui de Richet $(1892,1906)$. À nouveau, plusieurs versions de sa conférence vont circuler avec des nuances (Pernot 1964, note 23, 19-20), mais son engagement paraît ferme.

Toutefois, ces engagements institutionnels publics en faveur de la recherche psychique sont les plus proéminents connus. Par la suite, l'affinité intellectuelle se lira davantage dans les thématiques travaillées et à travers des correspondances et échanges plus intimes. Depuis une perspective française, on remarque que Bergson maintient une certaine distance avec les métapsychistes locaux, qu'il ne cite jamais, contrairement à leurs homologues de langue anglaise. Le docteur Hubert Larcher fera remarquer le parallélisme sous-jacent des thématiques élaborées par Bergson et les principaux psychistes français (Méheust 1997). Ces

4. Pernot $(1964,15)$ utilisait cette expression de façon plus générale en faisant de cette conférence "le Discours de la Méthode des 'sciences psychiques' " du point de vue de " tous les 'psychistes' ". Méheust (2005) vient préciser que l'approche quantitative s'appuyant sur les statistiques, déjà employée à l'époque, n’est pas plébiscitée dans le discours bergsonien. 
connexions intertextuelles sont néanmoins restées clandestines : "Tout se passe une fois de plus comme si Bergson, qui a baigné dans le climat intellectuel de la métapsychique, en avait tiré la quintessence, mais sans trop vouloir ou pouvoir l'avouer" (Méheust 1999, 250). Méheust repère même l'influence possible des thèses magnétistes et métapsychistes derrière le modèle bergsonien de l'esprit :

Résumons-nous. Une conscience qui, loin de se limiter à l'état vigile de l'homme adulte et civilisé comme l'enseigne depuis Descartes la philosophie occidentale, est susceptible de plusieurs niveaux de tension, de plusieurs niveaux de profondeur, qui correspondent chacun à un mode spécifique de rapport au réel ; un moi susceptible de se contracter sur lui-même et qui, se dessaisissant de l'extériorité, nous un nouveau rapport au réel ; une capacité d'intuition, fusion de l'instinct et de l'intelligence, qui permet de se transporter dans les choses, de sympathiser avec elles; une mémoire immense, infinie en droit, qui ne se totalise et ne se contracte sur elle-même qu'au niveau du moi profond ; une perception, qui s'étend en droit à l'univers entier, mais, en fait, se voit, comme la mémoire, circonscrite aux nécessités de l'action : tous ces thèmes bergsoniens font écho, de façon savante et épurée, aux faits, aux pratiques, aux spéculations que les magnétiseurs ont mis en circulation dans la culture depuis un siècle, et que les psychistes comme Myers ont prolongés et réélaborés (Méheust 1999, 248-249).

Or, sous les apparences officielles, Bergson a tissé des liens avec les métapsychistes français. Le 13 juin 1914, il dîne chez Richet avec onze autres convives : Eugène Osty, Joseph Maxwell, Arnaud de Gramont, Camille Flammarion, Jean-Charles Roux, Albert von Schrenck-Notzing, Jules Courtier, Cesare di Vesme, le physicien de Watteville, l'ingénieur Lemerle et le docteur Bourbon. Ce premier dîner-réunion dit « dîner du 13, " du fait de la date et du nombre de convives, se répétera tous les 13 du mois, intégrant de nouveaux chercheurs et préparant l'avenir de la métapsychique, notamment la fondation de l'Institut métapsychique international (IMI) en 1919 (Bergson 2011, 130, note 40 ; Evrard 2016).

Donnons encore deux dates saillantes pour situer la correspondance inédite que nous nous apprêtons à présenter. En 1932, les dernières pages des Deux sources de la morale et de la religion permettent au philosophe de conclure son œuvre sur les espoirs suscités par la métapsychique, ses " conséquences morales" (Madelrieux and Waterlot 2009, 277), car " c'est bien à la métapsychique, plus qu'au mysticisme proprement dit, qu'il confie le rôle de transformer l'humanité, de dilater sa vision du monde " (Méheust 1999, 253). Alors que cette solution « a toujours paru insolite » d'après Pernot $(1964,3)$, dans la Revue métapsychique publiée par l'IMI, un chroniqueur souligne que «nul n'a jamais exprimé avec plus de 
clarté, d'élégance et de concision, quels devaient être la place, le rôle, les difficultés et les conséquences de la Métapsychique " Quartier 1932, 226). Pernot (1964) conclut qu'on ne peut pas détacher ces quelques pages de l'ensemble de l'œuvre de Bergson car l'intérêt et la confiance qu'il accorde ici à la "science psychique " "se sont manifestés en d'autres circonstances et plongent des racines profondes dans sa philosophie" (Pernot 1964, 3). Bien qu'elle soit rarement commentée, la conclusion bergsonienne " n'est pas l'expression d'un intérêt passager et négligeable" (Pernot 1964, 11). Vladimir Jankélévitch $(2019,1)$ y lit le résumé d'un ouvrage que Bergson n'aura pas le temps d'écrire.

Enfin, le 12 février 1936, Bergson crédite les travaux sur la télépathie et la médiumnité du mathématicien Jean Labadié, par une lettre qui sera utilisée en préface (Labadié 1939, 7-8). Ces travaux, réalisés de manière isolée et dans une espérance survivaliste, prêteront largement le flanc à la critique qui s'emploiera à les faire passer pour " représentatifs » de la métapsychique (Boll 1994).

\section{L'expérience du Chanoine Monin}

Cette correspondance a été découverte dans les archives privées du docteur Hubert Larcher, à son ancien domicile de Saint-Paul de Vence. ${ }^{5}$ Médecin et licencié en philosophie, membre puis directeur de l'IMI de 1977 à 1995, Larcher a mené de nombreuses recherches dans le champ de la métapsychique, de la thanatologie et de la phénoménologie ascétique et mystique (Larcher 2013) qui l'ont conduit à entretenir une amitié puissante avec Jean Guitton (1901-1999). Ensemble, ils se rendaient chaque année en pèlerinage sur la tombe de Bergson à Garches. ${ }^{6}$ Guitton, philosophe et écrivain français, membre de l'Académie française, définissait l'IMI comme "l'Académie française de la recherche sur la nature de l'homme " et il confia à son (futur) directeur, le 8 mars 1971, les documents adressés le 19 avril 1935 par le Chanoine Monin (1873-1970) de Meaux, Curé de Jouarre, à Bergson, ainsi que la réponse de celui-ci. Guitton avait

5. Je remercie chaleureusement pour leur accueil Catherine et Olivier Larcher.

6. Pour l'anecdote, j'ai également découvert dans ces archives que lors d'une séance expérimentale privée avec la médium Hélène Bouvier, le 8 février 1968, les membres de la Commission de méthodologie de l'IMI, dirigés par René Poirier et Gabriel Marcel, également membres de l'Académie des sciences morales et politiques, eurent droit à une intervention non sollicitée de la part de "l'esprit de Bergson " qui affirma, à travers Bouvier, qu'il "vient pour tous » et qu'il " faut continuer vos recherches : vous aurez des résultats." 
récupéré ces documents en tant qu'exécuteur testamentaire de Bergson, et leur diffusion avait été rendu possible suite à la mort du Chanoine Paul Monin, devenu l'abbé Monin, ${ }^{7}$ survenue le 3 décembre 1970 à l'âge de 97 ans. Son légataire universel, M. le Chanoine Gabriel Durand, aumônier de l'Abbaye Notre Dame de Jouarre, avait accordé à Larcher, le 4 novembre 1971, l'autorisation de les publier en précisant que le Chanoine Monin n'avait d'abord fait connaître cette relation qu'à un cercle d'amis, «mais, à l'approche de sa mort, il souhaitait qu'elle fût répandue, notamment parmi ses confrères, estimant qu'ils pourraient y trouver le même encouragement qu'il en avait tiré lui-même. " Larcher avait ainsi préparé un dossier à partir de ces documents qu'il n'a apparemment jamais soumis à publication.

Le Chanoine Monin intitule son texte de quatre pages "Expérience mystico-psychanalytique du 8 mars 1835. "Il l'a envoyé à Bergson un mois plus tard et a reçu une réponse sans délai.

Le Chanoine revient d'abord sur son parcours de vie, ses quatre années passées en tant que supérieur au Petit Séminaire de Meaux, sa licence de philosophie inachevée, et son attitude a priori sceptique :

Je crois avoir l'esprit positif ; j'ai le goût des mathématiques, du détail précis, de la critique sévère des documents. A priori je reste sceptique devant tout récit de merveilleux. Je me suis intéressé, entre autres, aux ouvrages de psychopathologie de Pierre Janet $[\ldots]$

Il confesse ensuite avoir traversé plusieurs épreuves et être, à 60 ans, déprimé, maintenant de façon artificielle une allure enthousiaste. "Je suis déçu, ne voyant pas aboutir certaines réformes de tactique religieuse que j'espérais pouvoir faire prévaloir. »

Ces conduites le mènent vers un épuisement de sa foi, source de souffrance, dont il tentait de se défendre par des prières " pour retrouver la lumière, et, avec la lumière, un peu d'entrain et de joie dans mon ministère. »

Il en vient ensuite au récit de son expérience proprement dite, qui implique l'un de ses bons amis prêtre, le curé Fortin, plus âgé que lui de deux ans. Ils ne pouvaient se voir qu'une fois par an du fait de la distance entre leurs paroisses (environ 60 kilomètres). Quoi qu'il en soit, ils partageaient les mêmes convictions, les mêmes espoirs et les mêmes déceptions. Dans la nuit du 7 au 8 mars 1935, vers trois ou quatre heures du matin, le

7. L'abbé Monin a réalisé des travaux sur la doctrine de l'Abondance, opposée à celle du Capital, à laquelle il a commencé à s'intéresser en 1937, soit deux ans après son expérience (Monin 1939). 
Chanoine Monin se réveille et ressent une "ferveur extraordinaire. » Il peine à trouver un terme adéquat pour formuler cette " crise de ferveur, » " folie de ferveur, " " crise de béatitude, " " folie de béatitude " ... Il la décrit ainsi :

J'ai eu nettement l'impression d'être dominé par une influence extérieure à moimême, laquelle respectait ma liberté tout en me surélevant. Je faisais des actes d'amour intense, sentant bien que c'était une forme extérieure qui les produisait en moi, sentant bien que de moi-même je n'aurais jamais pu parvenir à cette hauteur et à cette intensité, gardant cependant conscience de les produire de mon propre mouvement, de ma pleine liberté : ces deux points de vue ne me paraissaient nullement contradictoires. J'avais l'impression que ce pouvait être une réponse de la miséricorde de Dieu à mes prières désolées des périodes précédentes. Les tentations contre la foi se sont évanouies. Je sentais qu'elles ne pouvaient plus mordre, puisque je voyais, j'expérimentais, — termes qu'il faut comprendre sans aucune intervention des sens.

Le chanoine commente encore cet état lui ayant permis de recouvrer sa foi. Puis il explique s'être rendormi "doucement. " Il ressentait encore les effets de cette joie à son lever, puis avec de moins en moins d'intensité dans la matinée, jusqu’à disparaître vers huit heures. Il dit en avoir gardé un "souvenir délicieux » qu'il consigne par écrit deux jours après pour se garantir des déformations possibles de sa mémoire.

Le même jour, vendredi 8 mars 1935 à midi, il reçoit du Chanoine Caudron, archiprêtre de Melun, un télégramme notifiant : "Ai grande douleur apprendre décès subit abbé Fortin curé Maincy. "Étant son légataire universel en plus d'être son ami, il fait son possible pour arriver au plus vite près du défunt, mais ne parvient à Melun qu'à 16h45. Il apprend alors l'horrible vérité : son ami n'avait pas succombé à une maladie, comme il le supposait, mais à deux coups de couteau assénés à la gorge pendant son sommeil, par sa domestique prise de folie qui s'était ensuite pendue. Prenant des renseignements, il apprend par le médecin légiste que la mort est survenue au milieu de la nuit et par section des veines jugulaires, c'està-dire une mort plus lente que si la carotide avait été coupée. Relevant cette coïncidence horaire, il conclut : "Je crois qu'il y a là une confirmation expérimentale de la doctrine à laquelle l'abbé Fortin et moi avons voué notre vie. »

Il termine sa lettre en tentant d'éliminer l'hypothèse de l'autosuggestion, affirmant n'avoir pas pensé à l'abbé Fortin avant de recevoir le télégramme et avoir plutôt envisagé un décès naturel qui pouvait être survenu une 
ou deux heures avant le télégramme. Ce vécu a eu sur lui un impact très significatif :

Pour moi personnellement, cette expérience, survenant après une épreuve de tentations contre la foi, barre la route à toute objection soi-disant scientifique, et vaut tous les traités d'apologétique du monde.

Examinons maintenant le commentaire qu'en fit Bergson :8

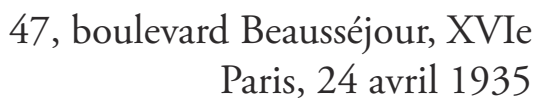

Monsieur le Curé,

Le récit que vous avez bien voulu me faire m'intéresse vivement. Depuis longtemps j'ai été amené à tenir pour réelle la " télépathie, " et plus particulièrement celle qui fait qu'on est averti de la mort d'un parent ou d'un ami au moment même où le fait se produit (quelle que soit la distance). Mais ce qu'il y a d'extraordinaire dans votre cas est la forme que l'avertissement a prise. Il semble que votre ami vous ait transmis quelque chose de ce qu'il éprouvait en quittant sa dépouille mortelle. Quelle expérience!

Conformément au vœu que vous exprimez, je garderai pour moi la communication que vous avez bien voulu me faire. D'ailleurs la chose va de soi. Une " auto-observation » ne doit jamais être publiée, ni même simplement mentionnée, sans l'autorisation expresse de l'auteur.

Avec tous mes remerciements je vous prie d'agréer, Monsieur le Curé, l'expression de mes sentiments respectueux.

H. Bergson

Il est étonnant de voir comment ce vécu est interprété selon le schème interprétatif classique des "fantômes de vivants " et de la télépathie. En effet, l'expérience de "ferveur extraordinaire » du Chanoine Monin

8. Nous avons travaillé à partir de la transcription de la lettre, dont l'original se trouve depuis 1972 dans les Archives départementales de Seine-et-Marne. MDZ1144). Paul MONIN (curé de Jouarre), «Expérience mystico-psychanalytique du 8 mars 1835 » [nuit de l'assassinat de l'abbé Fortin, curé de Maincy], récit daté 25 du 19 avril 1935 ; 11 p. mss. dact. et Biographie de l'abbé Paul Monin [par le chanoine Durand]. - 1 p. dact, avec une lettre autographe d'Henri Bergson (24 avril 1935). Ce dossier a été découvert à quelques semaines d'intervalle par Isabelle Rambaud (2019). 
n'évoque aucun décès ou circonstance négative, ni aucune information permettant d'identifier l'événement auquel elle pourrait être associée. Ce n'est que dans l'après-coup de cette mort spectaculaire que les deux sont combinés. De nombreux autres événements auraient pu compléter la signification de ce vécu venu remobiliser une foi altérée.

Dans sa lettre, Bergson va même plus loin que le chanoine en interprétant explicitement le cas comme une sorte de « dernière visite » ou d'avertissement du défunt. Cela pourrait correspondre à ce que Madelrieux et Waterlot (2009, 278) décrivent comme le « déplacement d'attention de la télépathie vers la médiumnité, " qui lui fournirait une manière scientifique de reconstruire une vision de l'au-delà, et notamment cette " vision d'au-delà dans une expérience scientifique élargie " (Bergson 1932, 338) à laquelle il entendait faire jouer un rôle civilisateur et moral (Pernot 1964, 2). Bergson associe cette crise de béatitude à un partage du vécu du mourant au moment où celui-ci quitte son corps, faisant écho aux expériences de mort imminente qui l'ont tant intéressé (Evrard 2018). L'ensemble du cas semble un juste retour aux Phantasms of the living (Gurney et al. 1886) ou "Fantômes de vivants " qui explique par la télépathie des phénomènes de communication et d'apparition lors de situations de crise.

En regard de la chronologie et de l'évolution des attitudes de Bergson par rapport aux recherches psychiques, cette lettre est la manifestation la plus limpide du fait que Bergson s'est convaincu de l'existence de la télépathie. Alors que depuis ses premières études de 1886, puis par ses nombreuses tergiversations dans ses conférences et ses textes, le philosophe manifestait une certaine prudence à l'égard de la réalité des phénomènes, il admet clairement ici " tenir pour réelle la 'télépathie' depuis longtemps. » Il ne s'agit plus d'encourager la quête épistémologique des sciences psychiques, ou de procéder à des paris philosophiques en misant sur des hypothèses, mais bien de se positionner sur le plan ontologique. À la question de Madelrieux et Waterlot (2009, 273), ${ }^{9}$ "Bergson y a-t-il cru ? " une réponse affirmative paraît justifiée. Ce matériel semble donner raison à Pernot $(1964,4)$ qui affirmait que le Bergson « observateur » des sciences psychiques en était devenu un " adepte. »

9. Malderieux, Sibertin-Blanc et Waterlot (2013, xxvii) vont même jusqu'à demander "Et jusqu’à quel point ? ". La question serait véritablement pertinente si Bergson avait précisément défini ce qu’il comprenait par «télépathie. " Méheust (1999), s’appuyant sur les témoignages de Jules Romains et Gabriel Marcel, interroge plutôt le fait que Bergson ait toujours nié les phénomènes de prémonition car ils ne s'intégraient pas dans sa théorie de la durée. 
On pourrait encore supputer une certaine complaisance de Bergson face à ce correspondant dont l'enthousiasme et la ferveur renouvelés le laissent difficilement accessible à un échange critique. Dans ces circonstances, Bergson a pu faire preuve de "sympathie " avec cet homme de foi et dépasser ses réserves habituelles. Cependant, Bergson ne prévoyant pas de publication de cet échange a pu aussi bien faire montre d'une parfaite sincérité. Comparativement, sa lettre ultérieure à Labadié (12 février 1936) laisse planer plus d'ambiguïtés quant à son positionnement personnel, puisqu'il mentionne, en observateur distant, les " faits déjà connus " et " ceux que vous avez observés vous-même » qui devraient emporter la conviction, sans confesser que sa propre opinion était déjà faite en ce qui concerne la télépathie dite " de crise. »

Une autre forme de complaisance revient à ne pas interroger la dimension libidinale de cette " expérience mystico-psychanalytique. " Cette " crise de béatitude » et ces « actes d'amour intenses » évoque une forme de jouissance autre, conceptualisée par Michèle Montrelay (1997) puis Lacan, jouissance non-phallique, dépassant l'ordre du signifiant, et entretenant un rapport fugace mais direct avec le Réel. Du fait de l'intitulé qu'il donne à son texte, le Chanoine Monin, par ailleurs progressiste, semblait faire partie de ceux qui, dans l'Église, s'ouvrait à cette " abomination moderniste " (Desmazières 2011). Pour autant, son expérience ne lui suscite que des échos dans le registre de la foi en évitant tout réductionnisme quant à cet état mystique.

\section{Conclusion}

La question de la réalité de la télépathie semble avoir été un objet de préoccupation pour Bergson depuis ses premières années en tant que professeur de philosophie à Clermont-Ferrand. Sur la fin de sa vie, ainsi que le montre cette lettre inédite, il a pu exprimer une pleine conviction quant à la réalité des phénomènes télépathiques, même si le terme forgé par Myers, maintenu entre guillemets, nécessitait toujours un affinage. Ces éléments connus du dossier n'offrent aucune certitude quant à la date de ce basculement, et aux raisons qui ont fait passer Bergson d'une position de scepticisme méthodique, puis de perplexité, à une adhésion intime, bien que dissimulée.

Tout au long de sa carrière, Bergson a maintenu une certaine ambiguïté quant à son positionnement par rapport aux recherches psychiques, s'avançant avec prudence et humilité dans ses constats, adaptant ses 
propos à leurs contextes de diffusion. Il a toutefois saisi à bras le corps ce domaine non seulement en lisant les travaux de ses contemporains, mais en expérimentant la suggestion mentale sur des sujets hypnotisés (sans succès), ainsi que les phénomènes physiques de la médiumnité avec le médium Eusapia Palladino (avec une conviction subjective plus forte). Ce double jeu a laissé des traces dans son héritage, de la même manière que pour Freud (Evrard et al. 2017 ; Fujita 2014 ; Trochu 2014). Les historiens ont collecté de nombreux indices de ce "secret de Polichinelle " (Méheust 1999, 240) d'un intérêt soutenu, fécond et stratégique de Bergson pour ces phénomènes aux marges de la science, à une époque où leur abord scientifique sentait déjà le souffre (Evrard 2016).

Désormais, deux tâches difficiles restent à accomplir pour les lecteurs de Bergson : la première consisterait à "re-penser » les travaux de Bergson à la lumière de ses positions en faveur de la réalité des phénomènes " anomaux " étudiés par les sciences psychiques de l'époque et la parapsychologie contemporaine. Le philosophe a-t-il véritablement développé son modèle des rapports entre l'esprit et la matière pour le rendre compatible avec la télépathie, comme l'ont supputé de nombreux chercheurs en parapsychologie (Kelly et al. 2007) ? La démonstration des " perceptions inconscientes » qu'il a faite dans Matière et mémoire (Bergson 2012, chapitre 1) semble également appliquée dans l'étude de la télépathie comme «débordement du mental sur le cérébral " (Madelrieux and Waterlot 2009, 287). Pernot (1964, 11-15) soutient que les différentes œuvres de Bergson donnent des clefs grâce auxquelles « on trouverait finalement l'explication des 'phénomènes psychiques', " à la fois télépathiques et spirites (Pernot $1964,17)$. Il y décrit progressivement une nouvelle vision de l'attention à la vie qui serait suffisamment puissante et dégagée de tout intérêt pratique qu'elle en deviendrait pure conscience, pure activité créatrice, en quelque sorte un effort pour accéder à un plan de vie spirituel, supérieur et détaché des vies biologique et psychologique. Pernot $(1964,12)$ postule que ces mouvements qui ne sont pas adaptatifs, ces actions qui ne sont pas " efficaces, » permettraient aux perceptions télépathiques de s'actualiser exceptionnellement sous forme de prolongements moteurs. On peut faire l'hypothèse inverse en considérant que son modèle dualiste et spiritualiste et son attention pour la parapsychologie sont deux entreprises différentes, ce qui est l'interprétation dominante d'une œuvre aux multiples influences. Mais pour Pernot $(1964,18)$, cette compatibilité entre spiritualisme et "spiritisme " chez Bergson tient à ce qu'il se réclame d'une attitude 
empirique ou expérimentale qui n’en font pas « un spiritualiste comme les autres " (Pernot 1964, 17).

La seconde tâche serait de tirer de Bergson des hypothèses de recherche fécondes (Freedman et al. 2018). Bergson, penseur de la "pénétration mutuelle, " discute à plusieurs reprises de "l'empiètement réciproque " des monades héritées de Leibniz, brisant cette vision enfermante d'entités finies sans réelles communications entre elles. Il compare les échanges qui pourraient s'accomplir entre diverses consciences aux phénomènes d'endosmose (Fujita 2014, 153), dans une interpénétration qui devrait répondre à des lois naturelles limitatives. Au-delà de ces métaphores suggestives, c'est le modèle du cerveau-filtre qui inspire actuellement le plus de recherches (Kelly et al. 2007 ; van Dongen 2014), notamment celles de l'équipe de Morris Freedman et ses collègues (2018) qui tentent d'identifier les zones cérébrales qui inhibent les facultés parapsychologiques. La porte entrouverte à la fin des Deux sources montre une direction à suivre ; reste à s'assurer que la recherche psychique peut effectivement se concilier avec le bergsonisme.

\section{Bibliographie}

Benrubi, Isaak. 1942. Souvenirs sur Henri Bergson. Paris : Delachaux \& Niestlé.

Bergson, Henri. 1886a. «De la simulation inconsciente dans l'hypnotisme.» Revue philosophique de la France et de l'étranger, 22 : 525-531.

Bergson, Henri. 1886b. Lettre à Frederic W.H. Myers du $1^{\text {er }}$ décembre 1886. Myers Papers. Trinity College. Cambridge.

Bergson, Henri. 1887. Lettre à Frederic W.H. Myers du 20 janvier 1887. Myers Papers. Trinity College, Cambridge.

Bergson, Henri. 2008. Les Deux sources de la morale et de la religion. Paris : Presses universitaires de France.

Bergson, Henri. 2012. Matière et mémoire : Essai sur la relation du corps à l'esprit. Paris : Presses universitaires de France.

Bergson, Henri. 2017. L'Energie spirituelle. Paris : Presses universitaires de France.

Bergson, Henri. 1972. Mélanges: L'idée de lieu chez Aristote, Durée et simultanéité, correspondance, pièces diverses, documents. Édité par André Robinet. Paris: Presses universitaires de France. 
Bergson, Henri. 2002. Correspondances. Édité par by André Robinet. Paris : Presses universitaires de France.

Bergson, Henri. 2011. Écrits philosophiques. Paris : Presses universitaires de France.

Boll, Marcel. 1944. L'Occultisme devant la science. Paris : Presses universitaires de France.

Courtier, Jules. 1908. Rapport sur les séances d'Eusapia Palladino à l'Institut général psychologique en 1905, 1906, 1907 et 1908. Paris : Institut général psychologique.

De Rochas, Albert. 1901. "Le Rêve. » Annales des sciences psychiques 10(3) : 160-72.

Desmazières, Agnès. 2011. L'Inconscient au paradis: comment les catholiques ont reçu la psychanalyse. Paris : Payot.

Evrard, Renaud. 2016. La Légende de l'esprit. Enquête sur 150 ans de parapsychologie. Escalquens : Trajectoire.

Evrard, Renaud. 2018. "L'Exaltation de la mémoire : une approche bergsonienne des expériences de mort imminente. "Intellectica 68 : 257-89.

Evrard, Renaud et Thomas Rabeyron. 2017. "La signification occulte des rêves : Freud pourfendeur ou pourvoyeur de mythes ? "Bulletin de psychologie, 70(6) : 463-476.

Freedman, Morris, Malcolm Binns, Fuqiang Gao, Melissa Holmes, Austyn Roseborough, Stephen Strother, Antonino Vallesi, Stanley Jeffers, Claude Alain, Peter Whitehouse, Jennifer D. Ryan, Robert Chen, Michael D. Cusimano, and Sandra E. Black. 2018. "MindMatter Interactions and the Frontal Lobes of the Brain : A Novel Neurobiological Model of psi Inhibition. " Explore 14(1) : 76-85.

Fujita, Hisashi. 2007. «Spiritualisme et spiritisme. L'hypnose, la télépathie, la recherche psychique dans la philosophie de Bergson. " Études de langue et littérature françaises (Société japonaise de langue et littérature françaises) $91: 168-183$

Fujita, Hisashi. 2014. "Télépathie : la recherche psychique de Bergson et la métapsychologie de Freud. " In Bergson et Freud, édité par Brigitte Sitbon, 141-54. Paris : Presses universitaires de France.

Grogin, Robert C. 1988. The Bergsonian Controversy in France, 1900-1914. Alberta : The University of Calgary Press. 
Gurney, Edmund, Frederic W.H. Myers, and Frank Podmore. 1886. Phantasms of the Living, 2 vol. London : Society for Psychical Research. James, William. 1890. Principles of psychology, vol. 2. New York : Dover. Jankélévitch, Vladimir. 2019. Henri Bergson. Paris : Presses universitaires de France.

Kelly, Emily F., Edward. W. Kelly, Adam Crabtree, Alan Gauld, Maurice Grosso, and Bruce Greyson. 2007. Irreducible Mind : Toward a Psychology for the $21^{\text {st }}$ Century. Lanham, MD : Rowman \& Littlefield.

Labadié, Jean. 1939. Aux frontières de l'au-delà, Choses vécues. Paris: Grasset.

Larcher, Hubert. 2013. L'Odyssée de la Conscience. Anthologie d'études sur la mort, la mystique et le paranormal par le Dr Hubert Larcher. Paris : Éditions de l'Institut Métapsychique International.

Luckhurst, Robert. 2002. The Invention of Telepathy. 1870-1901. Oxford : Oxford University Press.

Madelrieux, Stéphane, Guillaume Sibertin-Blanc, et Ghislain Waterlot. 2013. "Présentation. " In 'Le rêve' suivi de 'Fantômes de vivants' par Henri Bergson, xv-xxxv. Paris : Presses universitaires de France.

Madelrieux, Stéphane et Ghislain Waterlot. 2009. "'Fantômes de vivants' et 'recherche psychique'. "In L'Energie spirituelle, par Henri Bergson, 273-290. Paris : Presses universitaires de France.

Méheust, Bertrand. 1997. "À propos de métapsychique et de parapsychologie : entretien avec le Dr Hubert Larcher, par Bertrand Méheust. " Synapse, 141 :16-24.

Méheust, Bertrand. 1999. Somnambulisme et médiumnité. Tome 2 : Le choc des sciences psychiques. Paris : Institut Synthélabo.

Méheust, Bertrand. 2005. 100 mots pour comprendre la voyance. Paris : Les Empêcheurs de penser en rond.

Meunier, Georges. 1910. "Chez M. Bergson. » In Ce quils pensent du merveilleux, 81-107. Paris : Albin Michel.

Monin, Paul. 1939. "L'abondance et la religion. Conférence du 21 avril 1939. "Consulté en ligne le 05/06/2019 https://www. economiedistributive.fr/L-abondance-et-la-religion.

Montrelay, Michèle. 2001. Entretien avec Alain Didier-Weill. In Quartier Lacan, 181-96. Paris : Denoël.

Myers, Frederic W. H. 1887a. «On a Case of Alleged Hypnotic Hyperacuity of Vision. " Mind 12 : 154-156. 
Myers, Frederic W. H. 1887b. " Note on Certain Reported Cases of Hypnotic Hyperesthesia. " Proceedings of the Society for Psychical Research 4 : 532-39.

Myers, Frederic W. H. 1903. Human Personality and Its Survival of Bodily Death. London : Longmans, Green and co.

Nicolas, Serge, Anne Marchal, and Frederic Isel. 2000. « La psychologie au XIXème siècle. " Revue d'Histoire des Sciences Humaines 2(1) : 57-103.

Pernot, Camille. 1964. "Spiritualisme et spiritisme chez Bergson. » Revue de l'enseignement philosophique 15(3) : 1-23.

Plas, Régine. 2000. Naissance d'une science humaine: la psychologie. Les psychologues et le merveilleux psychique. Rennes: PUR.

Price, Henry H. 1941. "Obituary: Henri Bergson. " Proceedings of the Society for Psychical Research, 1940-1941, 44(164) : 271-276.

Quartier, Charles. 1932. "Recension de Les deux sources de la morale et de la religion. "Revue métapsychique, 13 : 222-26.

Rambaud, Isabelle. 2019. "Une lettre inédite d'Henri Bergson (24 avril 1935). Le chanoine, le prix Nobel et la télépathie. » Bulletin de la Société historique de Meaux et sa région 16 : 149-161.

Richet, Charles. 1884. «La suggestion mentale et le calcul des probabilités. » Revue philosophique de la France et de l'étranger 18 : 609-674.

Richet, Charles. 1892. "L'avenir de la psychologie. " Annales des sciences psychiques $2: 341-350$.

Richet, Charles. 1906. «L'avenir de la psychologie. » In Atti del V Congresso Internazionale di Psicologia, édité par Sante de Sanctis 166-173. Rome : Forzani.

Trochu, Thibaud. 2014. "Bergson et Freud : la 'recherche psychique' et l'exploration de l'inconscient'. "In Bergson et Freud, édité par Brigitte Sitbon, 197-215. Paris: Presses universitaires de France.

Sarafidis, Karl. 2009. «La 'rêverie' de Bergson. Métaphysique et spiritisme. » Alliage 65 : 73-86.

Van Dongen, Hein. 2014. "A machine for the making of Gods: On the meaning of 'filters' in Henri Bergson. » In Wild Beasts of the Philosophical Desert: Philosophers on Telepathy and Other Exceptional Experiences, édité par Hein van Dongen, Hans Gerding, and Rico Sneller, 92-109. Newcastle : Cambridge Scholars Publishing. 
Renaud Evrard est psychologue clinicien, Maître de conférences habilité à diriger des recherches en psychologie à l'Université de Lorraine. Il a cofondé le Centre d'information, de recherche et de consultation sur les expériences exceptionnelles. Il est l'auteur de plusieurs travaux sur les aspects cliniques, historiques, anthropologiques et sociologiques de l'étude du " paranormal. »

Résumé : L'intérêt de Henri Bergson (1859-1941) pour les recherches psychiques, également appelées parapsychologie ou métapsychique, est un " secret de Polichinelle " selon le sociologue et philosophe Bertrand Méheust (1999, 240). Il a déjà fait l'objet de nombreux commentaires. Bergson étant resté particulièrement prudent dans la mention de son intérêt, il est complexe de combiner les différentes pièces de ce puzzle pour donner une représentation conforme à la progression de sa pensée. La découverte dans les archives privées du docteur Hubert Larcher (1921-2008) d’une lettre inédite de Bergson au sujet de la télépathie est l'occasion de faire le point sur les connaissances acquises à ce sujet, afin de contextualiser cette correspondance et son potentiel apport théorique.

Mots-clés : télépathie, recherche psychique, Society for Psychical Research, hallucinations.

\begin{abstract}
Henri Bergson's (1859-1941) interest in psychical research, also known as parapsychology or metapsychics, is an "open secret" according to sociologist and philosopher Bertrand Méheust (1999, 240). It has already been the subject of many commentaries. Bergson remained cautious in mentioning his interest in these subjects. It is therefore difficult to combine the different pieces of this puzzle to give a representation in accordance with the progression of his thought. The discovery, in the private archives of Dr. Hubert Larcher (1921-2008), of an unpublished letter from Bergson on the subject of telepathy is an opportunity to review the knowledge acquired on this topic, in order to contextualize this correspondence and its potential theoretical contribution.
\end{abstract}

Keywords: telepathy, psychical research, Society for Psychical Research, hallucinations. 


